\title{
Bulk Electronic Structure of Metals Resolved with Scanning Tunneling Microscopy
}

\author{
J. I. Pascual, ${ }^{1}$ A. Dick, ${ }^{2,3}$ M. Hansmann, ${ }^{4}$ H.-P. Rust, ${ }^{4}$ J. Neugebauer, ${ }^{2,3}$ and K. Horn ${ }^{4}$ \\ ${ }^{1}$ Institut für Experimentalphysik, Freie Universität Berlin, Arnimallee 14, 14195 Berlin, Germany \\ ${ }^{2}$ Max-Planck-Institut für Eisenforschung, Max-Planck-Str. 1, 40237 Düsseldorf, Germany \\ ${ }^{3}$ Universität Paderborn, Warburger Str. 100, 33098 Paderborn, Germany \\ ${ }^{4}$ Fritz-Haber-Institut der Max-Planck-Gesellschaft, Faradayweg 4-6, 14195 Berlin, Germany
}

(Received 12 September 2005; published 1 February 2006)

\begin{abstract}
We demonstrate that bulk band structure can have a strong influence in scanning tunneling microscopy measurements by resolving electronic interference patterns associated with scattering phenomena of bulk states at a metal surface and reconstructing the bulk band topology. Our data reveal that bulk information can be detected because states at the edge of the surface-projected bulk band have a predominant role on the scattering patterns. With the aid of density functional calculations, we associate this effect with an intrinsic increase in the projected density of states of edge states. This enhancement is characteristic of the three-dimensional bulk band curvature, a phenomenon analog to a van Hove singularity.
\end{abstract}

DOI: 10.1103/PhysRevLett.96.046801

PACS numbers: 73.20.At, 68.37.Ef, 71.20.-b

The capabilities of the scanning tunneling microscope go far beyond imaging surfaces with atomic resolution by being able to probe the surface electronic structure, and extensive use has been made of this feature [1]. On metal surfaces, the electron dynamics can be analyzed by studying the characteristic wave patterns appearing in the proximity of surface defects such as steps and impurities [2-4]. In a general picture, the wave patterns are interpreted as the screening response of a $2 \mathrm{D}$ electron gas to defects such as steps or impurities. The wave patterns can be energy resolved by measuring spatial maps of the differential conductance $d I / d V$, which reproduce to first approximation the (energy dependent) local density of states (LDOS). In this case, the characteristic wavelength extracted from the $d I / d V$ oscillations can be easily related with the state's wave vector $\mathbf{k}$ by simply performing a Fourier transformation [5-7]. Standing electron waves have been extensively analyzed to locally resolve different surface states properties, such as electron dispersion [7,8], hot electron lifetimes $[9,10]$, or confinement at small terraces [11].

Bulk electronic states are also present at a metal surface, but their influence on the standing wave patterns and hence their detection by a surface probe is not immediately obvious [12-14]. They are thought to vanish near the termination of the bulk. Moreover, their projection onto the surface leads to a continuum band of states, which will suppress any oscillatory behavior of the density of states in real space. Here, we demonstrate that bulk band states influence standing wave patterns observed in STM. Our results reveal that states at the projected band edge have an unexpected predominant role, giving rise to a net oscillatory component in the proximity of surface defects, and allowing the reconstruction of a bulk band topology in reciprocal space using the Fourier-transform-STM technique. Density functional calculations confirm that band-edge states are intrinsically enhanced due threedimensional curvature of the band in the bulk.
For this study the (110) surface of a silver single crystal is most appropriate. This surface has projected bulk states around the $\bar{\Gamma}$ point in all the unoccupied energy region, which will tend to dominate the tunnel conductivity of a STM [Fig. 1(a)]. For larger wave vectors, the edge of the projected band limits with a bulk band gap centered at the boundary of the (110) surface Brillouin zone, at the $\bar{Y}$ point. The gap supports two surface states. The bottom of the highest lying state $S_{2}$ is located at $1.7 \mathrm{eV}$ above the Fermi energy $\left(E_{\mathrm{F}}\right)[7,15,16]$; the lowest state $S_{3}$ is partially occupied (onset energy lying at $\sim 50 \mathrm{meV}$ below $E_{\mathrm{F}}$ ), and extends up in energy until $\sim 200 \mathrm{meV}$ above $E_{\mathrm{F}}$, where it practically disappears from the surface region [17]. In the energy region between 0.3 and $1.7 \mathrm{eV}$ only states originating from bulk bands are present at the surface. Here, we focus our investigation in this energy region.

The experiments are performed on a custom-made scanning tunneling microscope (STM) working at $\sim 5 \mathrm{~K}$ [19]. At sample bias $V_{s}$, the $d I / d V$ is related to the local density of states at $E-E_{\mathrm{F}}=e V_{s}$. A lock-in technique is used to acquire the $d I / d V$ signal continuously, while the surface topography is explored. Figure 1(b) shows a stepped region of the $\operatorname{Ag}(110)$ surface. When $e V_{s}$ matches the energy of either the $S_{2}$ or the $S_{3}$ surface states the $d I / d V$ maps show standing wave patterns decaying away from the monatomic steps $[7,17,20]$. In Fig. 1(c) $e V_{s}$ lies, however, in the region where only bulk states exist. In the corresponding $d I / d V$ map clear oscillations can still be observed. Since there are no surface-related states in this energy range, we associate the origin of these oscillations with scattering processes of bulk states at the surface.

The behavior of the oscillation's wavelength with energy further supports this assignment. In Fig. 2(a) we plot a $d I / d V$ map as a function of energy (vertical axis) and distance along the [001] direction (horizontal axis), i.e., a $d I / d V\left(E, d_{[001]}\right)$ map. The data are acquired on the topside terrace of a monatomic step (located on the left). $d I / d V$ 
a)
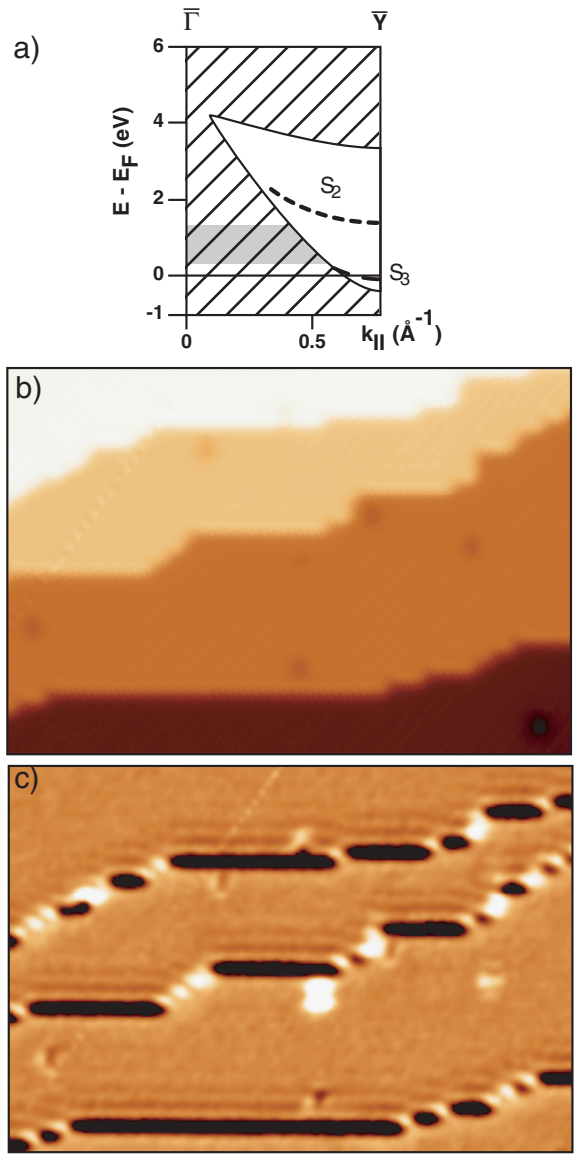

FIG. 1 (color online). (a) Model of $\mathrm{Ag}(110)$ band structure based on Refs. $[7,15,16]$. The white region corresponds to the band gap. The gray-shadowed region indicates the energy window between the two surface states. (b) STM topograph and (c) $d I / d V$ map of a stepped region of the $\operatorname{Ag}(110)$ surface. The $25 \mathrm{~nm} \times 16 \mathrm{~nm}$ map is measured with $V_{s}=1.6 \mathrm{eV}$, thus lying right below the onset of the $S_{2}$ surface state.

oscillations decay away from the step, and their wavelength increases with energy. Strong oscillations due to the onset of the $S_{2}$ surface state are seen at the top of Fig. 2(a). In Fig. 2(c) we show the result of a onedimensional Fourier transform for every constant energy $d I / d V$ cut in Fig. 2(a). For surface states, this procedure reconstructs the topology of electronic states in reciprocal space from real-space $d I / d V$ measurements [7]. Here it reproduces a feature whose dispersion and topology follows the edge of the projected $\mathrm{Ag}$ bulk bands present in this region. Therefore, we ascribe these $d I / d V$ oscillations to scattering processes of bulk electrons at the surface.

The origin of bulk-related oscillations is at this point not clear. At the bulk band edge (BBE), a sharp cutoff of the continuum of wave vectors may lead to a weak LDOS oscillation with wave vector $k_{\mathrm{BBE}}(E)$ around scattering centers [12]. This effect could qualitatively account for our observations. However, as we discuss below, a detailed analysis of resulting wave patterns at small terraces limited

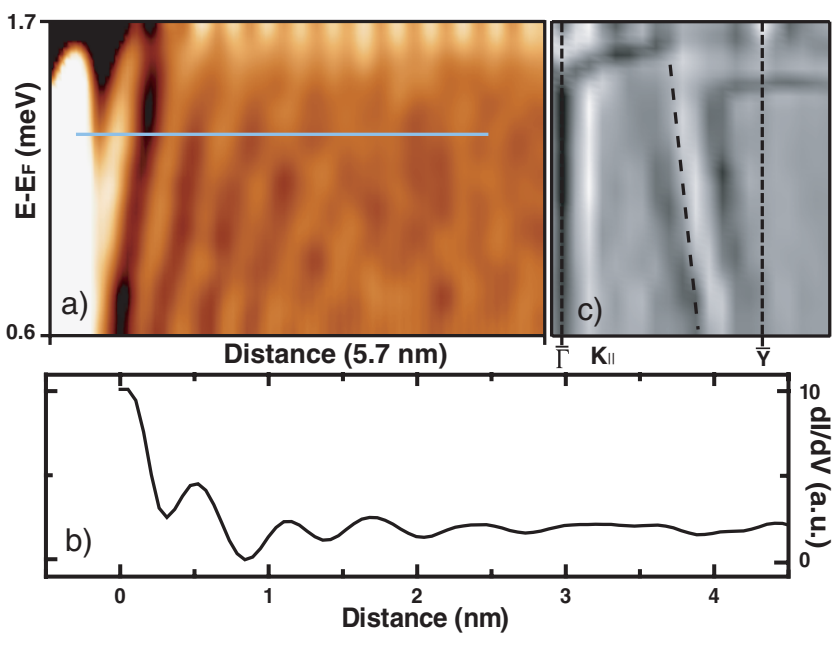

FIG. 2 (color online). (a) $d I / d V$ map of a $\operatorname{Ag}(110)$ region close to a step (bright feature left) as a function of the energy and the distance along the [001] direction. A few oscillations of $d I / d V$ are due to scattering of bulk states at the monatomic step. The oscillations disperse as the energy is increased. The strong increase of the signal at $E-E_{\mathrm{F}}=1.7 \mathrm{eV}$ is due to the onset of the $\operatorname{Ag}(110)$ surface state [7]. (b) $d I / d V$ profile along the line shown in (a). (c) One-dimensional Fourier transform of (a) as a function of the energy [vertical axis as in (c)]. A Laplace filter has been applied to emphasize non- $d c$ information. The dashed profile indicates the gap edge obtained by our DFT calculations, shown in Fig. 4.

by monatomic steps reveals a more subtle origin for these bulk band related features in the data.

At small terraces, the surface electronic states may become localized, as they are confined by monatomic steps. This gives rise to standing waves patterns with characteristics of a particle-in-a-box behavior [11]. The patterns then appear only at discrete energies, those at which the state's wavelength is commensurate with the length of the confined region (the so-called phase-addition rule). For the case of bulk states, their surface-projected components might also be confined at small terraces. Since the surface-projected band is composed of a continuous range of wave vectors, several of these may simultaneously fulfill the phase-addition rule, resulting in DOS oscillations composed of a discrete number of overlapping standing waves at a certain energy, each associated with one of the harmonics fitting into the terrace resonator. Moreover, since the bulk band is now discrete due to confinement, states at the band edge should not play a superior role in the resulting standing wave pattern.

This reasoning can be investigated on the basis of the data in Fig. 3, which shows a $d I / d V\left(E, d_{[001]}\right)$ map acquired onto a $\sim 36 \AA$ wide terrace terminated by two parallel descending steps. Both steps confine the wave function at the terrace region, creating standing wave patterns. In strong contrast to our reasoning above, single wavelength $d I / d V$ oscillations are found evolving with energy with discrete changes in number of nodes. Figure 3(c) shows several profiles from Fig. 3(b) taken at energy values 
with the largest $d I / d V$ oscillations' amplitude. There, the number of nodes decreases monotonously from 8 to 5 . At higher energies, bulk-related features cannot be detected due to the much larger intensity of the $S_{2}$ surface band. Overall, the pattern resembles that found similarly by Bürgi et al. [11] for confined Shockley surface states in $\operatorname{Ag}(111)$ narrow terraces; however, here it corresponds to bulk states.

The corresponding Fourier map [Fig. 3(d)] reveals the shape of the projected bulk band edge, albeit discretized because of the confinement on the terrace. Nodes appear at wave-vector values $6.8 \pm 0.3 \mathrm{~nm}^{-1}, \quad 5.9 \pm 0.2 \mathrm{~nm}^{-1}$, $5.1 \pm 0.2 \mathrm{~nm}^{-1}$, and $4.3 \pm 0.2 \mathrm{~nm}^{-1}$, from lower to higher energy. These values are consistent with a one-dimensional model where a particle is confined in a infinite potential box of width $37 \AA$. This width is in close agreement with the estimated size of the terrace [see model in Fig. 3(a)], thus indicating that surface atomic steps may show high
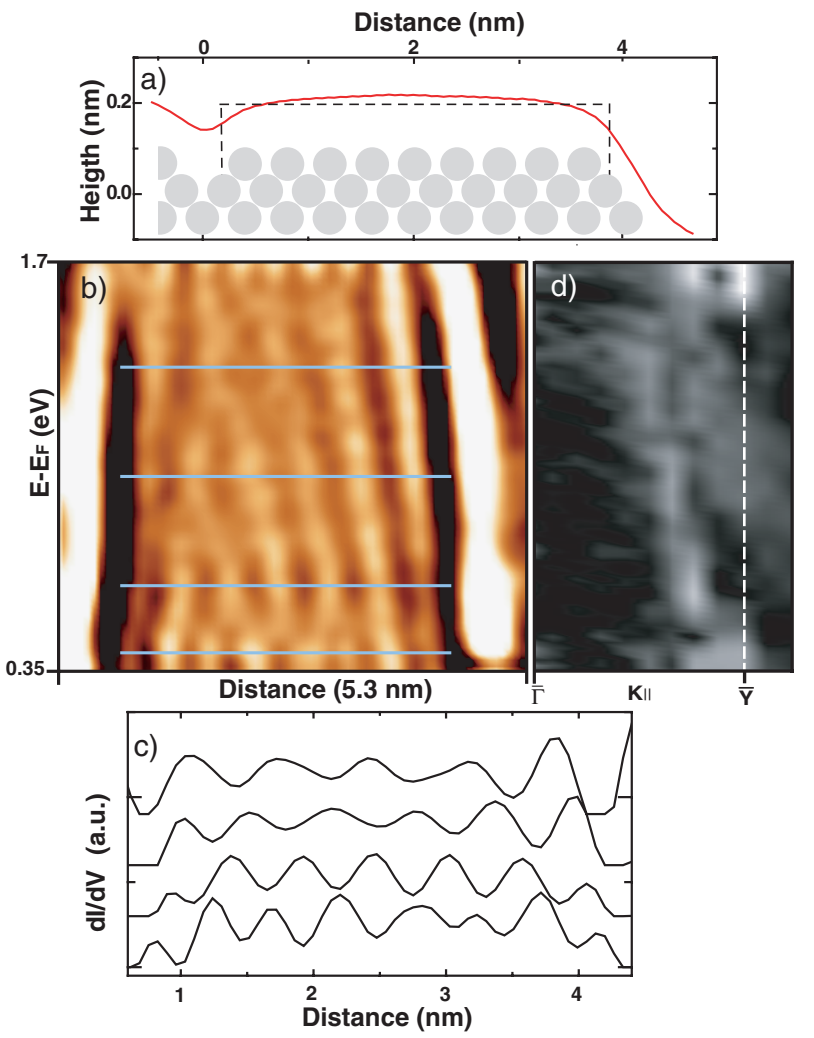

FIG. 3 (color online). $\quad d I / d V$ measurement on a $\sim 36 \AA$ terrace of $\operatorname{Ag}(110)$. (a) Height profile of the terrace along the [001] direction. Dashed lines indicate the size of a one-dimensional potential box model used to derive the wave-vector values found in (d). (b) $d I / d V$ signal in a color scale as a function of energy and real-space position along the [001] direction (cf. Fig. 2). Horizontal lines point at $d I / d V$ profiles shown in (c). They correspond to those energy values where the oscillations' amplitude is larger. A decreasing integer number of nodes is found. (d) $1 \mathrm{D}$ Fourier transform of (b). The real-space axis is transformed into reciprocal space. The positions in the surface Brillouin zone are indicated by the $\bar{\Gamma}$ and $\bar{Y}$ labels. The bulk band edge appears discretized due to confinement. reflectivity also for the scattering of bulk-related states. From the experiments we conclude that the edge of projected bulk bands has an enhanced weight at the surface over the rest of the bulk states. This is especially remarkable in this surface since band-edge states have larger wave vector than other states in the band, thus decaying faster into vacuum. The result is that at the surface the projected band behaves like a two-dimensional state whose dispersion draws the band edge, easing its observation with STM.

What is the origin of the LDOS enhancement at the surface band gap edge? In order to identify the causes for this phenomenon, we have performed ab initio calculations of the $\operatorname{Ag}(110)$ electronic structure. The surface has been modeled by a repeated slab geometry, with different thickness and with a vacuum region of $10 \AA$. Electronic structure and total energy for these structures have been calculated by employing plane-wave pseudopotential density functional theory (DFT) in the local-density approximation (LDA). A Troullier-Martins pseudopotential for Ag with the $4 d, 5 d$, and $5 p$ states as valence has been used [21]. The inclusion of silver $4 d$ valence states in our calculations is of fundamental importance since they are found to be responsible for the extension of bulk bands in the vacuum region and therefore essential for a correct description of the $\operatorname{Ag}(110)$ surface. The calculated surface band structure [Fig. 4(a)] is in excellent agreement with experiment and, in contrast to a previous ab initio study [16], reveals the presence of surface state $S_{3}$.

Our calculations [18] show that the projected bulk band in the experimentally relevant energy window is formed by two different types of bulk states that behave very differently in the vacuum region. One of them, composed mainly of $p$-like bulk states, quickly fades into the vacuum, having then a negligible weight there. The other band, with a predominantly $d$-orbital character, has considerable weight close to the surface. We focus therefore on this band [shown in Fig. 4(a) as thin solid lines] as it is responsible for the detected tunnel conductivity in the projected bulk band region. In Fig. 4(b) we plot the DOS of this band versus $k_{\|}$at constant energy. In agreement with experiment, a sharp increase in the density of states is found right at band edge, i.e., at $k_{\|}=k_{\mathrm{BBE}}$. In a three-dimensional picture of the band structure, the edge of projected bulk bands arises from the band curvature in the direction perpendicular to the surface. The band folding causes an enhancement of the number of states with wave vector $k_{\mathrm{BBE}}$, an effect conceptually similar to a van Hove singularity. The magnitude of this DOS enhancement is specific for this surface; however, such effect can be always associated with a projected band edge, and therefore, generalized to other metallic systems.

In STM, however, the probing of the surface electronic structure occurs in the vacuum at tip-surface separations of $\sim 5 \AA$, rather than directly at the surface. To account for the vacuum decay of bulk states we include in Fig. 4(b) an additional DOS versus $k_{\|}$plot for the same energy cut, but 


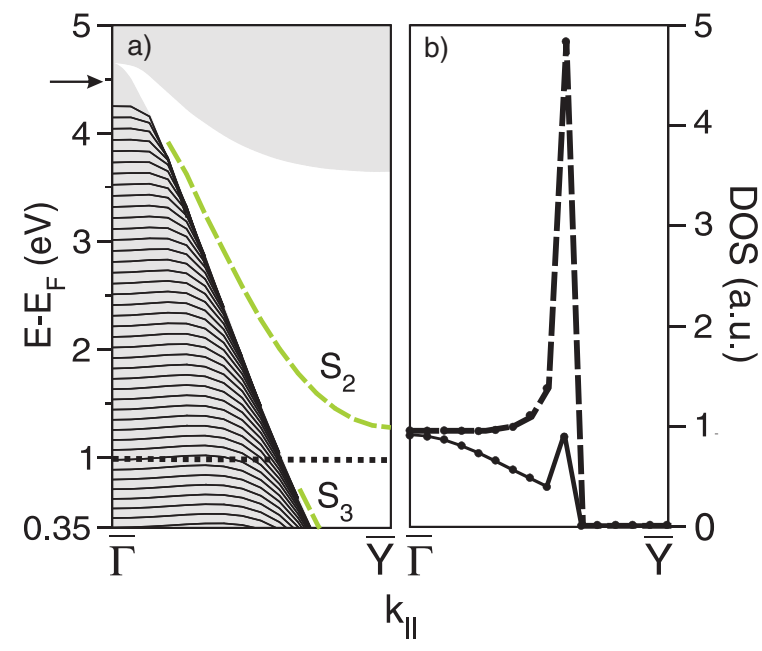

FIG. 4 (color online). (a) DFT results of the $\operatorname{Ag}(110)$ surface band structure: states with negligible [possessing DOS with weight less than 5\% of maximum contribution [18]] weight are hidden for clarity. Projected bulk bands (gray shaded area) and surface states (dashed green lines) reproduce available experimental data. Selected bulk states forming the projected bulk band structure for energies below the vacuum level are shown as thin black lines. (b) (Dashed line) Density of states as a function of $k_{\|}$along $\overline{\Gamma Y}$ for a constant energy $E=E_{\mathrm{F}}+1.0 \mathrm{eV}$ shown as a horizontal dotted line in (a). The enhancement of the DOS at the band edge is due to the folding of bulk bands along the surface normal. Solid line models the DOS behavior at $5.3 \AA$ above the surface, for the same constant energy cut. Gaussian smoothing with broadening of $0.05 \mathrm{eV}$ has been applied to each electronic state.

now obtained at a distance of $5.3 \AA$ above the surface. This height simulates a typical tip-sample distance. At this height the DOS discontinuity at the band edge still is present, in spite of these states having a larger wave vector $\left|k_{\|}\right|$and consequently stronger decay into the vacuum [12]. Band-edge states will give rise to interference patterns similar as (two-dimensional) Sockley surface states, thus accounting for the experimental observations.

In summary, we have shown that the density of bulk states near a surface experiences a van-Hove-like enhancement right at the band edge due to the three-dimensional band curvature. These band-edge states dominate over other bulk states in the scattering with surface defects, causing a net oscillatory component in the surface LDOS that can be detected with an STM and used to reconstruct the projected band topology in reciprocal space. Our results demonstrate that bulk states may have a relative strong weight in the last atomic layer, being sensitive to surface defects. Since the enhancement at the band edge appears to be a general phenomenon, the resolution of a bulk band topology with STM is not restricted to the metal surface shown here, but it can be observed in a large variety of metal surfaces [18].
K. H. gratefully acknowledges support by the European Union under the "Access to Research Infrastructures" program, and also support of the European Science Foundation EUROCORES program SONS under progamme MOLVIC through funds from the Deutsche Forschungsgemeinschaft, and the EC Sixth Framework program.

[1] Scanning Tunneling Microscopy, edited by R. Wiesendanger and H.-J. Guntherodt Springer Series in Surface Sciences Vol. 1-3 (Springer, New York, 1993).

[2] M.F. Crommie, C.P. Lutz, and D. M. Eigler, Nature (London) 363, 524 (1993).

[3] Y. Hasegawa and P. Avouris, Phys. Rev. Lett. 71, 1071 (1993).

[4] P. Avouris and I. W. Lyo, Science 264, 942 (1994).

[5] P. T. Sprunger, L. Petersen, E. W. Plummer, E. Laegsgaard, and F. Besenbacher, Science 275, 1764 (1997).

[6] L. Petersen, P. T. Sprunger, P. Hofmann, E. Laegsgaard, B. G. Briner, M. Doering, H. P. Rust, A. M. Bradshaw, F. Besenbacher, and E.W. Plummer, Phys. Rev. B 57, R6858 (1998).

[7] J. I. Pascual, Z. Song, J. J. Jackiw, K. Horn, and H. P. Rust, Phys. Rev. B 63, 241103(R) (2001).

[8] O. Jeandupeux, L. Burgi, A. Hirstein, H. Brune, and K. Kern, Phys. Rev. B 59, 15926 (1999).

[9] L. Bürgi, O. Jeandupeux, H. Brune, and K. Kern, Phys. Rev. Lett. 82, 4516 (1999).

[10] K. F. Braun and K. H. Rieder, Phys. Rev. Lett. 88, 096801 (2002).

[11] L. Bürgi, O. Jeandupeux, A. Hirstein, H. Brune, and K. Kern, Phys. Rev. Lett. 81, 5370 (1998).

[12] L. Petersen, P. Laitenberger, E. Laegsgaard, and F. Besenbacher, Phys. Rev. B 58, 7361 (1998).

[13] M. Schmid, W. Hebenstreit, P. Varga, and S. Crampin, Phys. Rev. Lett. 76, 2298 (1996).

[14] B. Koslowski, C. Dietrich, and P. Ziemann, Surf. Sci. 557, 255 (2004).

[15] B. Reihl, R. R. Schlittler, and H. Neff, Phys. Rev. Lett. 52, 1826 (1984).

[16] K. M. Ho, B. N. Harmon, and S. H. Liu, Phys. Rev. Lett. 44, 1531 (1980).

[17] Our DFT calculations find that shortly before crossing the bulk band edge, the LDOS of the $S_{3}$ surface state decreases substantially at the surface. This state yields essential bulk character and does not transform into $S_{3}$-derived surface resonance state [to be published in Ref. [18]]. In agreement with this, in STM experiments the standing wave pattern associated with the $S_{3}$ surface state is resolved, which suddenly disappears for sample bias above $200 \mathrm{mV}$.

[18] A. Dick et al. (to be published).

[19] H.-P. Rust, J. Buisset, E. K. Schweizer, and L. Cramer, Rev. Sci. Instrum. 68, 129 (1997).

[20] L. Petersen, B. Schaefer, E. Laegsgaard, I. Stensgaard, and F. Besenbacher, Surf. Sci. 457, 319 (2000).

[21] N. Troullier and J. L. Martins, Solid State Commun. 74, 613 (1990). 